\title{
Capacity Analysis of a Multi-Cell Multi-Antenna Cooperative Cellular Network with Co-Channel Interference
}

\author{
Xiaohu Ge, Senior Member, IEEE, Kun Huang, Cheng-Xiang Wang, Senior Member, IEEE, \\ Xuemin Hong, and Xi Yang
}

\begin{abstract}
Characterization and modeling of co-channel interference is critical for the design and performance evaluation of realistic multi-cell cellular networks. In this paper, based on alpha stable processes, an analytical co-channel interference model is proposed for multi-cell multiple-input multi-output (MIMO) cellular networks. The impact of different channel parameters on the new interference model is analyzed numerically. Furthermore, the exact normalized downlink average capacity is derived for a multi-cell MIMO cellular network with co-channel interference. Moreover, the closed-form normalized downlink average capacity is derived for cell-edge users in multi-cell multipleinput single-output (MISO) cooperative cellular networks with co-channel interference. From the new co-channel interference model and capacity formulas, the impact of cooperative antennas and base stations on cell-edge user performance in the multicell multi-antenna cellular network is investigated by numerical methods. Numerical results show that cooperative transmission can improve the capacity performance of multi-cell multi-antenna cooperative cellular networks, especially in a scenario with a high density of interfering base stations. The capacity performance gain is degraded with the increased number of cooperative antennas or base stations.
\end{abstract}

Index Terms-Interference modeling, capacity analysis, multiple-input multiple-output (MIMO), cooperative transmission, co-channel interference.

Manuscript received August 31, 2010; revised March 12, 2011 and July 3, 2011; accepted August 29, 2011. The associate editor coordinating the review of this paper and approving it for publication was C. Yang.

X. Ge, K. Huang, and X. Yang are with the Department of Electronics and Information Engineering, Huazhong University of Science and Technology, Wuhan 430074, Hubei, P. R. China (e-mail: xhge@mail.hust.edu.cn, \{kunhuang, reneyangxi\}@smail.hust.edu.cn).

C.-X. Wang (corresponding author) is with the Joint Research Institute for Signal and Image Processing, School of Engineering and Physical Sciences, Heriot-Watt University, Edinburgh EH14 4AS, UK (e-mail: chengxiang.wang@hw.ac.uk).

$X$. Hong is with the School of Information Science and Technology, Xiamen University, Xiamen 361005, Fujian, China (e-mail: xuemin.hong@xmu.edu.cn).

The authors would like to acknowledge the support from the RCUK for the UK-China Science Bridges Project: R\&D on (B)4G Wireless Mobile Communications. X. Ge, K. Huang, and X. Yang also acknowledge the support from the National Natural Science Foundation of China (NSFC) (Grant No.: 60872007), the National 863 High Technology Program of China (Grant No.: 2009AA01Z239), and the Ministry of Science and Technology (MOST), China, International Science and Technology Collaboration Program (Grant No.: 0903). C.-X. Wang acknowledges the support from the Scottish Funding Council for the Joint Research Institute in Signal and Image Processing with the University of Edinburgh, as part of the Edinburgh Research Partnership in Engineering and Mathematics (ERPem), and the support by the Opening Project of the Key Laboratory of Cognitive Radio and Information Processing (Guilin University of Electronic Technology), Ministry of Education.

Digital Object Identifier 10.1109/TWC.2011.11.101551

\section{INTRODUCTION}

$\mathbf{T}$ O achieve high transmission data rates, multi-antenna technology has widely been adopted in the 3rd generation $(3 \mathrm{G})$ and 4 th generation $(4 \mathrm{G})$ mobile communication systems [1]. The multi-antenna technology can improve the system capacity proportionally with the minimum number of antennas at the transmitter and receiver in a single cell communication system [2]. However, the system capacity of multi-cell cellular networks is greatly degraded by the co-channel interference even with multiple antennas at the transmitters and receivers [3], [4]. As a result, co-channel interference modeling and capacity analysis of multi-cell multi-antenna cellular networks are of great importance in the next generation mobile communication systems.

Numerous interference models have been proposed in the literature for wireless communication systems [5]-[11]. Sousa proposed that the infinite aggregated interference from the homogeneous Poisson field of interferers can be modeled by an alpha stable distribution [5]. Furthermore, based on [5], an analytical expression for the instantaneous and second order distributions of the interference was presented in [6]. Salbaroli and Zanella investigated the interference characteristic function in a finite Poisson field of interferers in [7]. The cochannel interference statistics in a Poisson field of interferers was derived from a unified framework in [8]. Whereas, most models proposed in [5]-[8] were constrained to single antenna or single cell communication systems and did not fully consider the complex effects of wireless channels. For example, the interference models proposed in [5], [6] are only suitable for single antenna communication systems without considering shadowing and fading effects in wireless channels. The interference models proposed in [7], [8] did not consider multi-cell multi-antenna systems. Different from these existing models in [5]-[8], in this paper we propose an interference model for multi-cell multi-antenna cellular networks considering the effects of pathloss, shadowing, and small-scale fading in wireless channels. For multi-cell interference statistics, Gulati and Evans introduced a mathematical framework for the characterization of network interference in wireless networks in which interferers are scattered according to a spatial Poisson process and subject to path loss, shadowing, and multi-path fading [9]. Moreover, a unified framework from which the cochannel interference statistics in a Poisson field of interferer distributed on a parametric circular annular region was derived 
in [10]. Based on wider range of interferer topologies and Poisson-Poisson cluster field of interferers extended from [10], the applicability of the symmetric alpha stable and Gaussian mixture (with Middleton Class A as a particular form) distributions in modeling co-channel interference signal phase was demonstrated in [11]. In [10], [11], a unified framework for deriving interference models for various wireless network environments was developed and corresponding characteristic functions of interference signal phase models were illustrated. Based on the aforementioned results, we further derive an analytical co-channel interference signal power model for multi-cell multi-input multi-output (MIMO) cellular networks with the Poisson spatial distribution of interferers.

For the capacity analysis of multi-antenna cellular networks, many studies have been carried out [12]-[18]. Telatar and Foschini carried out the initial research for the point-topoint multi-antenna communication system and indicated that the system capacity increases linearly with the minimum number of antennas in the transmitter and receiver over uncorrelated flat Rayleigh fading channels [2], [12]. Furthermore, Webb presented a way of explicitly examining the effect of interference on the MIMO subchannel gains in a mutli-antenna communication system and derived asymptotic lower bounds on capacity with many interferers and in high interference-to-noise ratio [13]. Wang derived an exact closedform expression of the moment-generating function (MGF) of mutual information of MIMO channels with interference for a cellular network. Then, an exact expression of the MIMO ergodic capacity with the MGF formula of mutual information was proposed for Rayleigh fading channels [14]. Treating interference as noise was shown to be sum capacity achieving for the two-user single-input single-output (SISO) Gaussian interference channel in a low interference regime. Moreover, the low interference regime for the multi-input single-output (MISO) Gaussian interference channel was characterized in [15]. Kwak analyzed the capacity of MIMO channels in the presence of both antenna correlation and co-channel interference [16]. For the special case of separable correlations, Kwak derived analytical expressions for the key statistical properties of the spectral efficiency achievable with an arbitrary input covariance. Under a single cell communication system, Chiani developed an analytical framework to characterize the capacity of MIMO communication systems and derived the ergodic mutual information for MIMO systems in the presence of multiple MIMO co-channel interferers and noise [17]. Based on the mutual information of a MIMO system with co-channel interference, Ye revealed that equalpower interferers give worse performance than unequal-power interferers and a smaller number of interferers each with larger power degrades performance less than a larger number of interferers each with lower power [18].

Cooperative transmission in wireless communications has been studied in the recent literature [19]-[21]. Based on a pathloss channel model, Chen proposed an intuitive method for calculating the system diversity level in the generalized distributed antenna system with cooperative users [19]. Furthermore, a novel channel representation for a two-hop decentralized wireless relay network was proposed and analyzed considering the cooperative transmission [20]. For the cell-edge users, the capacity with beamforming cooperative transmission was investigated for the soft handover region in multi-cell MIMO cellular networks [21].

However, in all the aforementioned capacity studies, only simple scenarios, such as a single cell with finite interfering transmitters, were considered and the underlying channel models were limited to simple flat Rayleigh fading channels. Besides, the exact normalized average capacity of multi-cell MIMO cellular networks with co-channel interference has not been investigated. Moreover, detailed investigation of the analytical co-channel interference model used for multi-cell multi-antenna cellular networks is surprisingly rare in the open literature. Many existing cooperative transmission literature simply treat co-channel interference as noise [19]-[21].

Motivated by the above gaps, in this paper we derive the exact downlink average capacity of multi-cell MIMO cellular network with co-channel interference. The contributions and novelties of this paper are summarized as follows.

1) We propose an analytical co-channel interference model for multi-cell MIMO cellular networks with a Poisson spatial distribution of interfering transmitters, taking into account fading and shadowing effects in wireless channels.

2) From the proposed co-channel interference model, we derive the exact downlink average capacity of multi-cell MIMO cellular networks with co-channel interference.

3) The closed-form normalized downlink average capacity for cell-edge users in multi-cell MISO cooperative cellular networks with co-channel interference is derived for numerical analysis.

4) We study the normalized capacity of the multi-cell MISO cooperative cellular networks in great details and present some interesting observations.

The remainder of this paper is outlined as follows. Section II proposes the new analytical co-channel interference model in multi-cell MIMO cellular networks. In Section III, from the proposed co-channel interference model, the exact downlink average capacity of multi-cell MIMO cellular network is derived. Furthermore, a closed-form normalized downlink average capacity for cell-edge users in a multi-cell MISO cooperative cellular network with co-channel interference is obtained. Simulation results and analysis are presented in Section IV. Finally, conclusions are drawn in Section V.

\section{Co-Channel Interference Modeling And PERFORMANCE ANALYSIS}

\section{A. General Interference Model}

In this paper, interference analysis is focused on the downlink of cellular networks. A cell of cellular network is analogous to the geometrically based single-bounce macrocell model, where the scatters representing user equipments (UEs) are uniformly distributed within a circle centered at the base stations (BS). To simplify the system model used for interference analysis, we only consider effective interferers in our general interference model. As shown in Fig. 1, there are only two types of nodes. One type of nodes is the signal receiver noted as UE with $N_{r} \in[1, \cdots, \infty)$ antennas and the other type of nodes is the interfering transmitter, i.e., 


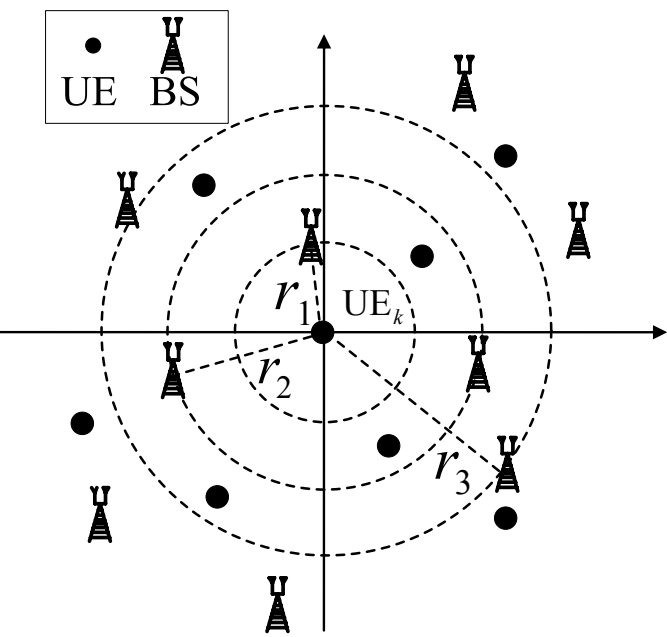

Fig. 1. General interference model.

BS with $N_{t} \in[1, \cdots, \infty)$ antennas. In idealized cellular networks, the BS locations are assumed to have a regular hexagonal structure. However, in real cellular networks, the BS locations are carefully chosen and optimized to take into account various practical factors such as building heights, user density, terrain features, etc. This typically leads to irregular and seemingly randomized BS locations, which can be well captured by Poisson distributions [22]. This Poisson model has widely been adopted in cellular networks to characterize BS locations [23], [24]. The Poisson model also provides theoretical tractability and scalability. Therefore, in this paper the locations of all BSs are also assumed to follow a Poisson spatial distribution in a two-dimensional infinite plane. Moreover, only one BS is assumed to exist in one cell. This general interference model can be used to describe the interference signals in multi-cell MIMO cellular networks.

\section{B. Interference Model of Multi-cell MIMO Cellular Networks}

In the aforementioned general interference model, as illustrated in Fig.1, every signal including the interference signal passes through an independent wireless channel, which means every signal is subject to independent path loss, shadowing, and Nakagami-m fading effect [25]. In general, the shadowing effect can be characterized by lognormal distributions. To simplify the calculation, the PDF of signal with shadowing effect can be approximated by a Gamma distribution [26]:

$$
p(x)=\frac{1}{\Gamma(\lambda)}\left(\frac{\lambda}{\Omega}\right)^{\lambda} x^{\lambda-1} e^{-\frac{\lambda}{\Omega} x}, x>0
$$

with

$$
\begin{gathered}
\Gamma(\lambda)=\int_{0}^{\infty} t^{\lambda-1} e^{-t} \mathrm{~d} t \\
\Omega=P_{r} \sqrt{(\lambda+1) / \lambda} \\
\lambda=1 /\left(e^{\left(\sigma_{d B} / 8.686\right)^{2}}-1\right)
\end{gathered}
$$

where $\sigma_{d B}$ is the shadow spread parameter expressed in decibels whose value usually ranges from 4 to 9 in practice and $P_{r}$ is the received average signal power at the receiver.
Considering that the shadowing effect in the wireless channel is approximated by the Gamma distribution, the wireless channel with Gamma shadowing and Nakagami-m fading can be further approximated by the Generalized-K $\left(K_{G}\right)$ distribution [27].

Without loss of generality, we select one of the users $\mathrm{UE}_{k}$ $(k=1, \cdots, \infty)$ as the signal receiver interfered by interfering transmitters from the given region in Fig. 1. This $\mathrm{UE}_{k}$ receives aggregated interference from the interfering transmitters, i.e., $\mathrm{BSs}$, in the given region. The aggregated interference at $\mathrm{UE}_{k}$ can be expressed as follows

$$
P^{R_{X}}=\sum_{b=1}^{\infty} \frac{I_{b}}{r_{b}^{\sigma_{r}}}=\sum_{b=1}^{\infty} \frac{\sum_{i=1}^{N_{r}}\left(I_{b, i}\right)}{r_{b}^{\sigma_{r}}}=\sum_{i=1}^{N_{r}}\left(\sum_{b=1}^{\infty} \frac{I_{b, i}}{r_{b}^{\sigma_{r}}}\right)
$$

where $I_{b}$ is the interference signal power received by $\mathrm{UE}_{k}$ from the BS $b$ without the path loss effect, $I_{b, i}$ is the interference signal power received by the antenna $i$ of $\mathrm{UE}_{k}$ from the BS $b$ without the path loss effect, $r_{b}^{-\sigma_{r}}$ is the path loss variable with path loss coefficient $\sigma_{r}$ and path distance $r_{b}$ from the BS $b$ to the user. The spatial distribution of BSs is a Poisson distribution with a density parameter $\lambda_{B S}$. Thus, the distribution of the aggregated interference at $\mathrm{UE}_{k}$ is governed by an alpha stable distribution [8] and expressed by the characteristic function [7],[8].

$$
\Phi_{P R x}(j w)=\exp \left(-|c w|^{\alpha}\left[1-j \operatorname{sign}(w) \tan \left(\frac{\pi \alpha}{2}\right)\right]\right)
$$

with

$$
\begin{gathered}
\operatorname{sign}(w)= \begin{cases}1, & w>0 \\
0, & w=0 \\
-1, & w<0\end{cases} \\
\alpha=\frac{2}{\sigma_{r}} \\
c=\sqrt[\alpha]{\lambda_{B S} q \mathbb{E}\left(I_{b}^{\alpha}\right)} \\
q= \begin{cases}\pi \Gamma(2-\alpha) \cos (\pi \alpha / 2) /(1-\alpha), & \alpha \neq 1 \\
\pi^{2} / 2, & \alpha=1\end{cases}
\end{gathered}
$$

where $\mathbb{E}(\cdot)$ is the expectation operator, $\Gamma(\cdot)$ is the Gamma function which is defined by (1b), $\alpha$ is the characteristic exponent, and $c$ is the scale parameter.

In Fig. 1, an interfering transmitter, i.e., a BS, with $N_{t}$ antennas has $N_{t}$ interference sub-streams from the closed space source and every antenna of $\mathrm{UE}_{k}$ can receive $N_{t}$ interference sub-streams from a BS. The path loss and shadowing effect for one BS-UE pair is assumed the same across all the antennas. The transmission power of every antenna is assumed to be equal and normalized to 1, i.e., $P_{a n t}=1$. Therefore, in multicell MIMO cellular networks, $I_{b}$ in (3d) is the sum of the interference signal power transmitted by the $N_{t}$ interference sub-streams from the same BS $b$ without the path loss effect. Furthermore, $I_{b}$ is expressed by 


$$
\begin{aligned}
I_{b} & =P_{a n t} w_{b}\left(\sum_{i=1}^{N_{r}} \sum_{j=1}^{N_{t}}\left|z_{b, i, j}\right|^{2}\right) \\
& =w_{b}\left(\sum_{i=1}^{N_{r}} \sum_{j=1}^{N_{t}}\left|z_{b, i, j}\right|^{2}\right)
\end{aligned}
$$

where $w_{b}$ is a random variable of Gamma shadowing process, which corresponds to the signal passing through the Gamma shadowing channel from the $\mathrm{BS} b$, and $z_{b, i, j}$ is the random variable of Nakagami-m fading process, which corresponds to the signal passing through the Nakagami-m fading channel from the transmitting antenna $j$ of BS $b$ to the receiving antenna $i$ of $\mathrm{UE}_{k}$.

Because the wireless channel with Gamma shadowing and Nakagami-m fading can be approximated by the $K_{G}$ distribution, the PDF of $I_{b}$ can be further derived as follows based on the Generalized-K random process theory [26]:

$f_{I}(y)=\frac{2\left(\frac{m \lambda}{\Omega}\right)^{\frac{N_{t} N_{r} m+\lambda}{2}}}{\Gamma\left(N_{t} N_{r} m\right) \Gamma(\lambda)} y^{\frac{N_{t} N_{r} m+\lambda-2}{2}} K_{\lambda-N_{t} N_{r} m}\left(2 \sqrt{\frac{m \lambda y}{\Omega}}\right)$

where $m$ is the Nakagami shaping factor, $K_{v}(\cdot)$ is the modified Bessel function of the second kind with order $v, \Omega$ and $\lambda$ are defined in (1c) and (1d), respectively.

In order to derive a closed-form expression for the PDF, we can perform an inverse Fourier transform on the characteristic function of (3a). For this purpose, a closed-form expression of (3d) should be first derived. Therefore, from (5) the following transform parameter $\gamma$ is derived accounting for the transformation in [28]

$$
\gamma=c^{\alpha}=\lambda_{B S} q\left(\frac{m \lambda}{\Omega}\right)^{-\alpha} \frac{\Gamma(\lambda+\alpha) \Gamma\left(N_{t} N_{r} m+\alpha\right)}{\Gamma\left(N_{t} N_{r} m\right) \Gamma(\lambda)} .
$$

Furthermore, we substitute (6) into (3d) and perform the inverse Fourier transform on the characteristic function of (3a). Ultimately a new PDF expression of the aggregated interference $P^{R_{X}}$ at the user in the multi-cell MIMO cellular networks is derived as

$$
f_{P R x}(y)=\frac{1}{2 \pi} \int_{-\infty}^{+\infty} \Phi_{P R x}(j w) \exp (-2 \pi j w y) \mathrm{d} w
$$

where $\Phi_{P R x}(j w)$ is given by (3a) with

$$
c=\left(\lambda_{B S} q\left(\frac{m \lambda}{\Omega}\right)^{-\alpha} \frac{\Gamma(\lambda+\alpha) \Gamma\left(N_{t} N_{r} m+\alpha\right)}{\Gamma\left(N_{t} N_{r} m\right) \Gamma(\lambda)}\right)^{\frac{1}{\alpha}} .
$$

When the path loss coefficient is configured as $\sigma_{r}=4$ which corresponds to the urban macro-cell with rich scattering environment [25], an analytical interference model is given by

$$
f_{P^{R x}}(y)=\sqrt{\gamma^{2} / 2 \pi} \frac{e^{-\gamma^{2} / 2 y}}{y^{3 / 2}}, y>0
$$

with

$$
\gamma=\lambda_{B S} \sqrt{2} \pi \Gamma\left(\frac{3}{2}\right)\left(\frac{m \lambda}{\Omega}\right)^{-\alpha} \frac{\Gamma\left(\lambda+\frac{1}{2}\right) \Gamma\left(N_{t} N_{r} m+\frac{1}{2}\right)}{\Gamma\left(N_{t} N_{r} m\right) \Gamma(\lambda)}
$$

where $\Gamma(\cdot), \Omega$ and $\lambda$ are denoted by (1b), (1c) and (1d), respectively.

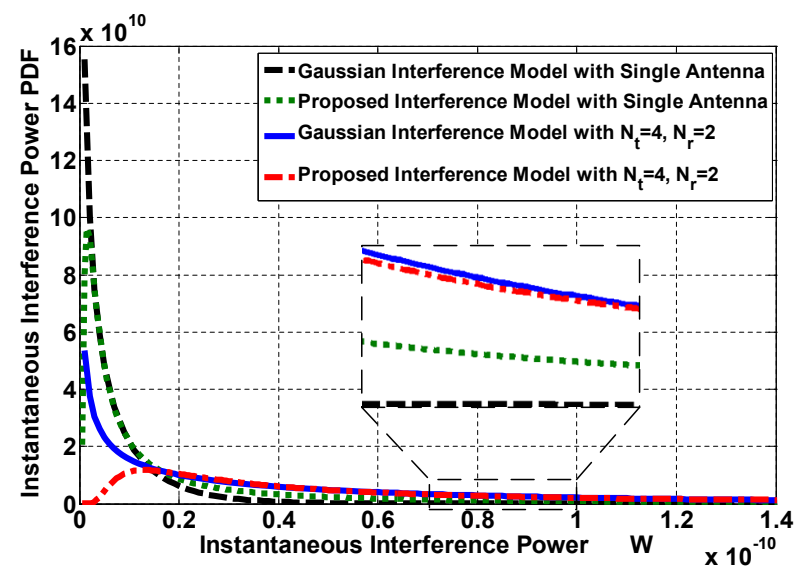

Fig. 2. Comparison of the instantaneous interference power PDFs of the proposed interference model and Gaussian interference model.

\section{Performance Analysis}

Based on the proposed new interference model, some performance evaluations can be numerically analyzed in detail. In the following analysis, some parameters of the interference model in Fig. 1 are configured as follows: $\sigma_{d B}=6$, which usually ranges from 4 to 9 in practice [25]; $m=1$, which usually ranges from 0.5 to 10 in practice [25]; $\sigma_{r}=4$, which corresponds to an urban macrocell with a rich scattering environment [25]; $N_{t}=4$ and $N_{r}=2$ [29]; the effective radius of interfering BS is assumed as 800 meters and the transmission power of interfering BS is assumed as $P_{r}=1 \mathrm{watt}$; the interfering BSs' density parameter is $\lambda_{B S}=1 /\left(\pi \times 800^{2}\right)$. In the previous work [11], [30], [31], Gaussian interference models were widely used. Based on some default parameters, in Fig. 2 we compare the instantaneous interference power PDFs of the proposed interference model with those of the Gaussian interference model. Both single antenna and multi-antenna scenarios were considered. In the single antenna scenario, the receiver and the interfering transmitters are all equipped with one antenna. In the multi-antenna scenario, the receiver has $N_{r}=2$ antennas and the interfering transmitters have $N_{t}=4$ antennas. For better visualization, the tail parts of the PDFs of two interference models are enlarged and compared in Fig. 2. It is found that the PDF of the proposed interference model has an obvious heavier tail compared with that of the Gaussian interference model. According to the stable distribution theory [32], [33], the heavy tail characteristic indicates that small probability events or rare events (e.g., interference with high power) may have a non-negligible impact on the alpha stable distribution. Therefore, the aggregate interference in multicell MIMO cellular networks can sometimes be dominated by individual high-power interfering signals. Moreover, in Fig. 3 we compare the instantaneous interference power cumulative distribution functions (CDFs) calculated by the numerical method and Monte-Carlo (MC) simulations. A good match is found between the two CDF curves, demonstrating the correctness of our derivations.

Furthermore, we analyze the impact of some parameters on the proposed interference model in Figs. 4-7. In Fig. 4, the probability of instantaneous interference power within the range from 0 to $2.5 \times 10^{-11}$ watt decreases with the increase 


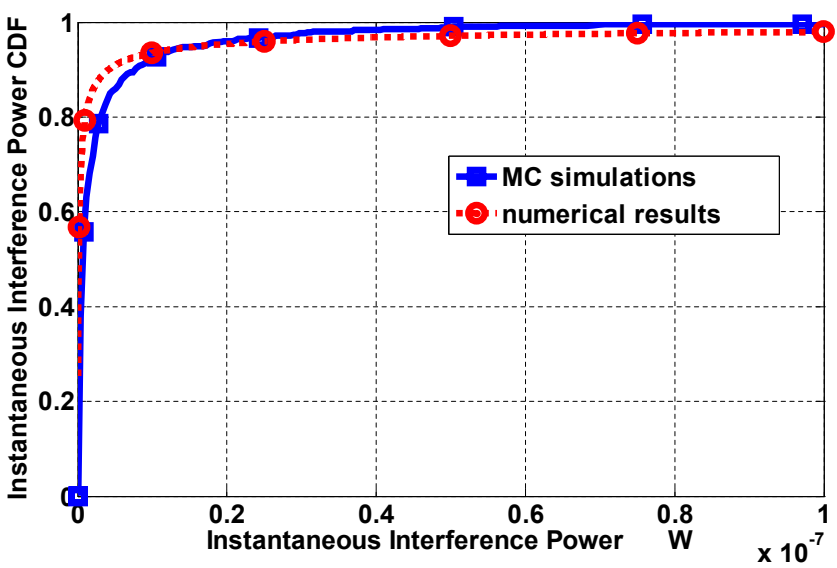

Fig. 3. Comparison of numerical results and $\mathrm{MC}$ simulations for the instantaneous interference power CDFs.

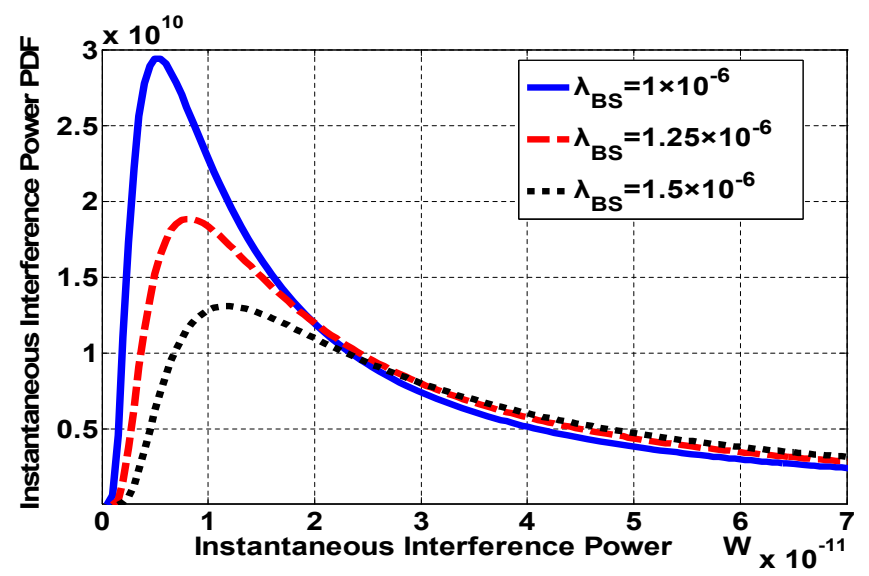

Fig. 4. Impact of the density parameter $\lambda_{B S}$ of base stations on the PDF of the aggregated interference.

of the BS density parameter $\lambda_{B S}$. When the instantaneous interference power exceeds $2.5 \times 10^{-10}$ watt, the probability of instantaneous interference power increases with the increase of $\lambda_{B S}$. Fig. 5 illustrates that when the instantaneous interference power is less than $0.8 \times 10^{-10}$ watt, the PDF increases with the increase of the path loss coefficient parameter $\sigma_{r}$. The probability of instantaneous interference power gradually decreases with the increase of $\sigma_{r}$ when the instantaneous interference power is larger than $0.8 \times 10^{-10}$ watt. Fig. 6 demonstrates that the probability of instantaneous interference power decreases with the increase of the number of transmission antennas $N_{t}$ per interfering transmitter when the instantaneous interference power is less than $0.12 \times 10^{-10}$ watt. If the instantaneous interference power exceeds $0.12 \times 10^{-10}$ watt, the trend is reversed. In Fig. 7, the probability of instantaneous interference power decreases with the increase of the number of receiving antennas $N_{r}$ per user when the instantaneous interference power is smaller than $0.3 \times 10^{-10}$ watt. After this turning point, the probability of instantaneous interference power gradually increases with the increase of $N_{r}$. Based on Figs. 4-7 and the alpha stable process theory [32], [33], we observe that these four parameters can significantly influence the burstiness of the aggregated interference in multi-cell MIMO cellular networks.

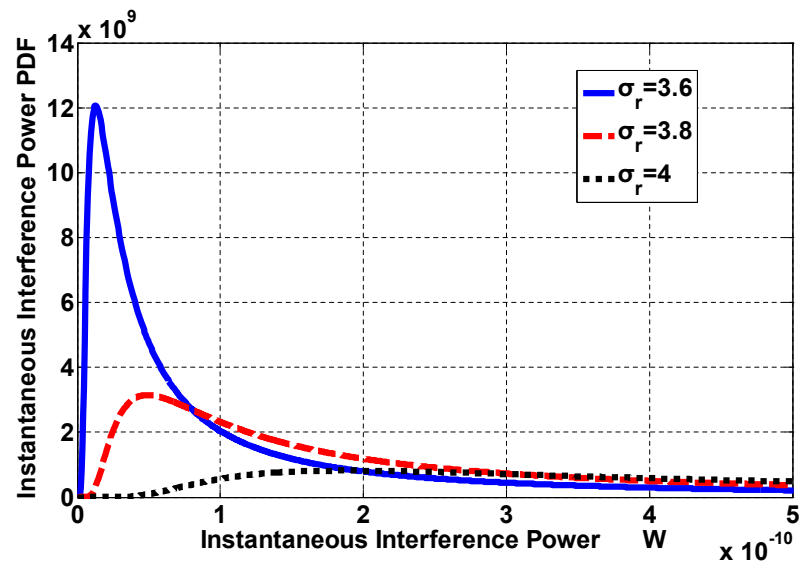

Fig. 5. Impact of the path loss coefficient parameter $\sigma_{r}$ on the PDF of the aggregated interference.

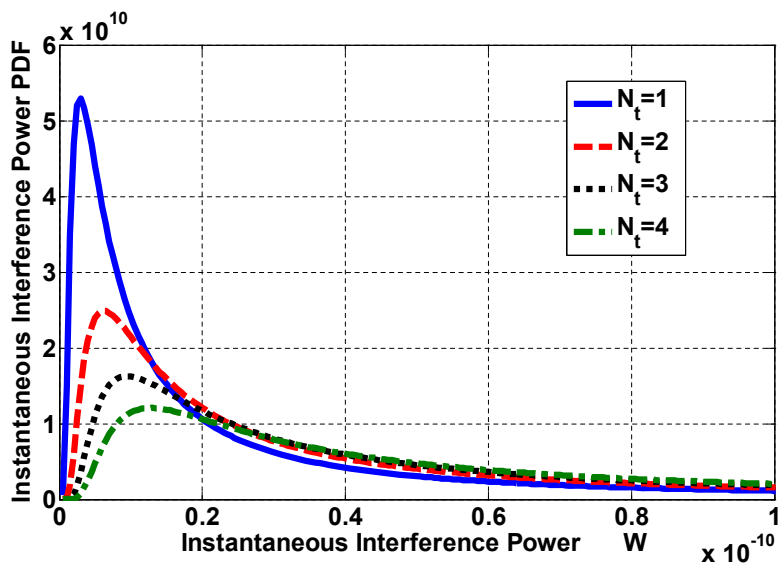

Fig. 6. Impact of the number of transmission antennas per interfering transmitter $N_{t}$ on the PDF of the aggregated interference.

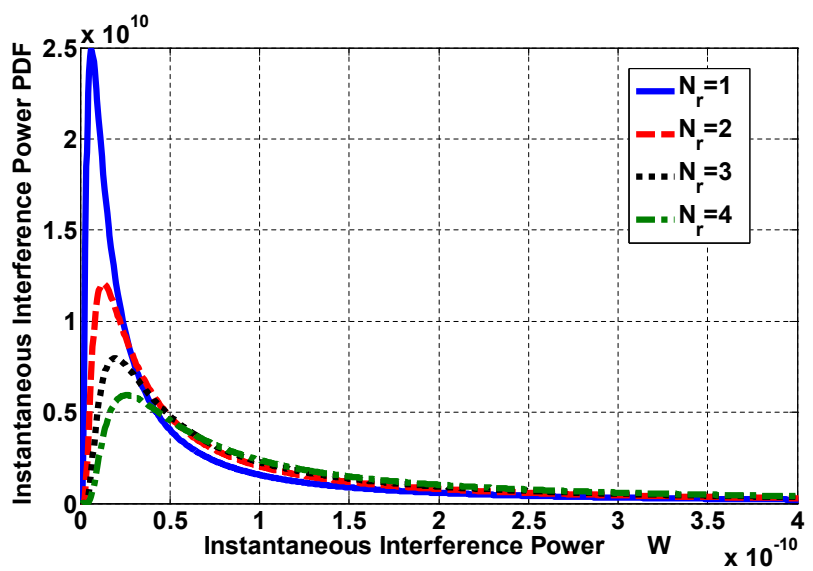

Fig. 7. Impact of the number of receiving antennas per user $N_{r}$ on the PDF of the aggregated interference.

\section{Capacity of Multi-Cell Mimo Cooperative Cellular Networks}

\section{A. Cooperative System}

Based on the proposed interference model, we further investigate the capacity of a multi-cell MIMO cooperative cellular network. The multi-cell MIMO cellular network considered in this paper is as follows: in the two dimensional given region, 


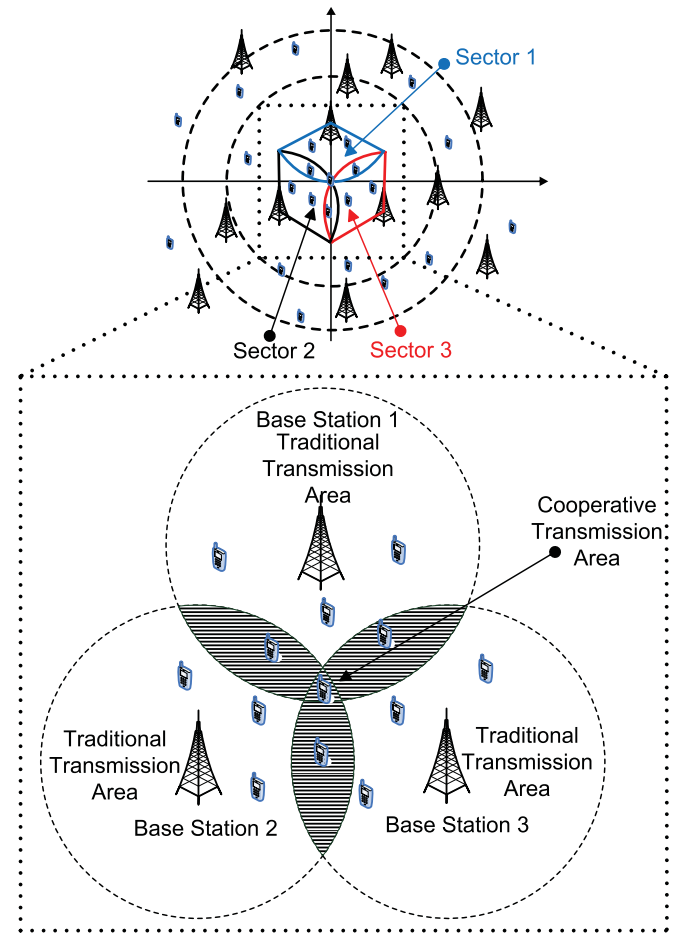

Fig. 8. A cooperative system with co-channel interference.

there are infinite BSs with $N_{t}$ antennas and user terminals with $N_{r}$ antennas. The location of interfering BSs is assumed to follow a Poisson spatial distribution. Every cell only has one BS. Without loss of generality, the three closest BSs are selected for cooperative transmission and the three closest BSs are located in a triangle structure. Furthermore, every BS is assumed to have three sectors and three adjacent sectors in different BSs are configured as a hexagon cooperative cluster structure as shown in Fig. 8. Therefore, the three closest BSs are called the cooperative BSs and these three adjacent sectors are called a cooperative cluster. In the following simulations, the number of cooperative BSs is not fixed to three and we further analyze the cooperative transmission performance with the number of cooperative BSs changing from 1 to 3 . The cooperative transmission is limited in the overlapping areas of the cells and this area is called cooperative transmission area. The rest of the area is called traditional transmission area. To explicitly describe the cooperative cluster structure, a detailed cooperative cluster structure with three cooperative BSs is zoomed in Fig. 8. To simplify the analysis, our capacity analysis of multi-cell MIMO cellular networks is limited to a cooperative cluster. Perfects channel state information (CSI) in the cooperative cluster is assumed to be available for every cooperative BS [21]. Considering that the interference has great impact on the cell-edge users in the multi-cell MIMO cellular network, this paper focuses on the downlink capacity of cell-edge users in the cooperative transmission area. In the cell-edge overlapping area, $K$ users are assumed to simultaneously receive the desired signal from the cooperative BSs $N_{b} \in[1,3]$ and the number of other users in this multicell MIMO cellular network is denoted as $K^{\prime}$. In the following numerical analysis, different numbers of cooperative BSs $N_{b}$ correspond to different cooperative transmission solutions.

\section{B. Downlink Capacity of Multi-cell MIMO Cooperative Cel- lular Network}

For analytical tractability, all wireless channels in the multicell MIMO cellular network are assumed to be Nakagami$\mathrm{m}$ fading channels with path loss. This assumption has been adopted in many wireless communication studies (e.g., [34][38]) and a recent study [39] has shown that shadowing effect does not cause major changes in the capacity PDF. Without considering cooperative transmission, the signal $y_{k}$ received by the cell-edge user $\mathrm{UE}_{k}$ can be expressed as [17]

$$
\begin{aligned}
y_{k} & =\sum_{c b=1}^{N_{b}} \mathbf{h}_{c b, k, c} \mathbf{x}_{c b, k, c}+\sum_{c b=1}^{N_{b}} \mathbf{h}_{c b, k, c} \sum_{j=1, j \neq k}^{K} \mathbf{x}_{c b, j, c} \\
& +\sum_{b=1}^{\infty} \mathbf{h}_{b, k, \bar{c}} \sum_{j=1}^{K^{\prime}} \mathbf{x}_{b, j, \bar{c}}+\mathbf{n}_{0} .
\end{aligned}
$$

On the right side of $(9 a)$, the first term is the expected signal for the $\mathrm{UE}_{k}$, the second term is the aggregated interference signal from the cooperative cluster $c$, the third term is the aggregated interference signal from the cluster $\bar{c}$, where $\bar{c}$ is the complement cluster of the cluster $c$, i.e., one of all other clusters excluding the cooperative cluster $\bar{c}$, and the fourth term $\mathbf{n}_{0}$ is the additive white Gaussian noise (AWGN) in the wireless channel. Where $\mathbf{h}_{c b, k, c}$ is the channel matrix between the cooperative $\mathrm{BS} c b$ and the user $\mathrm{UE}_{k}$ in the cooperative cluster $c, \mathbf{x}_{c b, k, c}$ is the signal vector from the cooperative BS $c b$ to the user $\mathrm{UE}_{k}$ in the cooperative cluster $c, \mathbf{h}_{b, k, \bar{c}}$ is the channel matrix between the user $\mathrm{UE}_{k}$ and the interfering $\mathrm{BS} b$ in the cluster $\bar{c}, \mathbf{x}_{b, k, \bar{c}}$ is the signal vector from the interfering BS $b$ in the cluster $\bar{c}$ to the user $\mathrm{UE}_{k}$ in the cluster $c$.

Considering the maximum ratio transmission / maximum ratio combining (MRT/MRC) approaches used in multi-cell MIMO cellular networks [40], [41], the interference in the cooperative cluster is eliminated and the signal $y_{k}$ received by the cell-edge user $\mathrm{UE}_{k}$ can be expressed as

$$
y_{k}=\sum_{c b=1}^{N_{b}} \mathbf{h}_{c b, k, c} \mathbf{x}_{c b, k, c}+\sum_{b=1}^{\infty} \mathbf{h}_{b, k, \bar{c}} \sum_{j=1}^{K^{\prime}} \mathbf{x}_{b, j, \bar{c}}+\mathbf{n}_{0} .
$$

Without changing the nature of the problem, we simply assume that all the weighted coefficients used for receive antennas in the MRT approach are equivalent. Moreover, the aggregated interference from different receive antennas is assumed to be statistically independent. In this case, the interference power from different receive antennas can be simply summed together, as shown in [40]. Therefore, the signal-to-interference-and-noise ratio (SINR) received by the user $\mathrm{UE}_{k}$ can be expressed by

$$
\mathrm{SINR}_{k}=\frac{P_{\mathrm{ant}} \lambda_{\max }\left(\mathbf{H}_{k, c} \mathbf{H}_{k, c}^{H}\right)}{N_{0}+\sum_{b=1}^{\infty} I_{b}}
$$

with

$$
\mathbf{H}_{k, c}=\left[\mathbf{h}_{1, k, c}, \mathbf{h}_{2, k, c}, \cdots, \mathbf{h}_{N_{b}, k, c}\right]
$$

where $P_{\text {ant }}$ and $I_{b}$ are the transmission power of each antenna and the sum of the interference signal power transmitted by 
the $N_{t}$ interference sub-streams from the same interfering BS $b$ outside the cooperative cluster $c$, respectively; $\mathbf{H}_{k, c}$ is the cooperative channel matrix in cooperative cluster $c$, which is composed by the sub-channel matrix from cooperative BSs to the user $\mathrm{UE}_{k}, \lambda_{\max }\left(\mathbf{A A}^{H}\right)$ is the maximum singular value of the matrix $\mathbf{A} \mathbf{A}^{H}$.

Based on the Shannon theory, the capacity of the interference channel linking user $\mathrm{UE}_{k}$ in the multi-cell MIMO cellular network can be expressed by

$$
C_{k}=\mathrm{B}_{\mathrm{w}} \log _{2}\left[1+\frac{P_{\mathrm{ant}} \lambda_{\max }\left(\mathbf{H}_{k, c} \mathbf{H}_{k, c}^{H}\right)}{N_{0}+P_{\mathrm{ant}} \sum_{b=1}^{\infty} \frac{1}{r_{b}^{\sigma_{r}}} w_{b}\left(\sum_{i=1}^{N_{r}} \sum_{j=1}^{N_{t}}\left|z_{b, i, j}\right|^{2}\right)}\right]
$$

where $B_{w}$ is the bandwidth in the wireless link. Considering that the power of AWGN $N_{0}$ can be ignored comparing to the power of the received interference signal [20], the average capacity of multi-cell MIMO cellular network can be given by

$$
C_{\text {Aver }} \simeq \mathrm{B}_{\mathrm{w}} \int_{0}^{\infty} \log _{2}(1+\eta) f(\eta) \mathrm{d} \eta
$$

with

$$
\eta=\frac{S_{d}}{S_{I}}=\frac{P_{\mathrm{ant}} \lambda_{\max }\left(\mathbf{H}_{k, c} \mathbf{H}_{k, c}^{H}\right)}{P_{\mathrm{ant}} \sum_{b=1}^{\infty} r_{b}^{-\sigma_{r}} w_{b}\left(\sum_{i=1}^{N_{r}} \sum_{j=1}^{N_{t}}\left|z_{b, i, j}\right|^{2}\right)}
$$

where $S_{d}$ and $S_{I}$ are the powers of the expected signal and aggregated interference signal, respectively. When the power of expected signal and aggregated interference signal are assumed to be statistically independent, the PDF of $\eta$ is derived as

$$
f(\eta)=\int_{0}^{\infty} f_{P R x}(z) f_{d}(\eta z) z \mathrm{~d} z
$$

where $f_{P R x}(z)$ is the PDF of aggregated interference signal and $f_{d}(\eta z)$ is the PDF of expected signal. Considering an urban macro-cell with rich scattering environment, i.e., $\sigma_{r}=$ 4 , let us substitute (8a) and (13) into (12a). Finally, a new exact downlink average capacity of multi-cell MIMO cellular network with co-channel interference is derived as

$$
\begin{aligned}
C_{\text {Aver }} & =\mathrm{B}_{\mathrm{w}} \sqrt{\frac{\gamma^{2}}{2 \pi}} \int_{0}^{\infty} \log _{2}(1+\eta) \\
& \times\left(\int_{0}^{\infty} e^{-\gamma^{2} / 2 z} z^{-1 / 2} f_{d}(\eta z) \mathrm{d} z\right) \mathrm{d} \eta
\end{aligned}
$$

where $\gamma$ is given by (8b). If only one BS transmitting expected signals in the cooperative cluster $c$ exists, (14a) is simplified as a downlink average capacity of point-to-point MIMO system with co-channel interference by (14b) and (14c)

$$
C_{\text {Aver }}=\mathrm{B}_{\mathrm{w}} \int_{0}^{\infty} \log _{2}\left(1+\gamma_{k}\right) f\left(\gamma_{k}\right) d \gamma_{k}
$$

with

$$
\gamma_{k}=\frac{P_{\text {ant }}}{P_{\text {ant }} \sum_{b=1}^{\infty} r_{b}^{-\sigma_{r}} w_{b}\left(\sum_{i=1}^{N_{r}} \sum_{j=1}^{N_{t}}\left|z_{b, i, j}\right|^{2}\right)} \lambda_{\max }\left(\mathbf{h}_{1, k, c} \mathbf{h}_{1, k, c}^{H}\right) .
$$

\section{Closed-form Downlink Capacity of Multi-cell MISO Cel- lular Network}

Because it is easier to integrate multiple antennas into the BSs than the user terminals in practice, in this section we further derive a new closed-form downlink average capacity for cell-edge users in a multi-cell MISO cooperative cellular network with co-channel interference.

In a cooperative cluster, one user can simultaneously receive the desired signals and interfering signals transmitted from cooperative BSs. To simplify our derivation, the time/frequency division multiple access (T/FDMA) or some such orthogonal strategies are assumed to be used in the cooperative intra-cluster, i.e., the multi-user interference within a single cooperative cluster is assumed to be negligible. Moreover, the power transmitted by every antenna in one BS is normalized to 1 , i.e., $P_{\text {ant }}=1$. To calculate the downlink capacity of multi-cell MISO cellular network, we should first get the signal covariance transmitted to the user $\mathrm{UE}_{k}$ by different BSs in the cooperative cluster $c$, which has been derived in the Appendix and is expressed by

$$
\mathbf{R}_{x x}=P_{\text {ant }} \sum_{c b=1}^{N_{b}} \sum_{j=1}^{N_{t}^{c}} \frac{1}{r_{c b}^{\sigma_{r}}}\left|z_{c b, j}\right|^{2}=\sum_{c b=1}^{N_{b}} \sum_{j=1}^{N_{t}^{c}} \frac{1}{r_{c b}^{\sigma_{r}}}\left|z_{c b, j}\right|^{2}
$$

where $N_{t}^{c}$ is the number of cooperative antennas transmitting the desired signal and $r_{c b}$ is the distance between the cooperative BS $c b$ and a cell-edge user.

For a single cell MISO cellular network, the downlink capacity of user $\mathrm{UE}_{k}$ is typically presented as follows [42]

$$
C_{k}=\mathrm{B}_{\mathrm{w}} \log _{2}\left(1+\frac{\mathbf{R}_{x x}}{N_{0}+P^{R_{X}}}\right)
$$

where $P^{R_{X}}$ is the aggregated interference.

Based on the assumption in the Appendix, the interfering signals and the desired signals from different cooperative BSs in the cooperative cluster $c$ to the user $\mathrm{UE}_{k}$ are assumed to be uncorrelated. Ideally, the interference within the cooperative cluster can be cancelled completely. Therefore, from the proposed interference model in (2), the aggregated interference in a multi-cell MISO cellular network is further expressed as follows

$$
P^{R_{X}}=P_{\text {ant }} \sum_{b=1}^{\infty} \sum_{j=1}^{N_{t}} \frac{1}{r_{b}^{\sigma_{r}}}\left|z_{b, j}\right|^{2}=\sum_{b=1}^{\infty} \sum_{j=1}^{N_{t}} \frac{1}{r_{b}^{\sigma_{r}}}\left|z_{b, j}\right|^{2}
$$

where $r_{b}$ is the distance between the interfering BS $b$ and the cooperative cluster and the locations of interfering BSs are governed by the Poisson spatial distribution, $z_{b, j}$ is a random variable following the Nakagami-m distribution and represents a signal passing through the Nakagami-m fading channel from antenna $j$ at interfering $\mathrm{BS} b$. 
Furthermore, we substitute (15) and (17) into (16). Considering the path loss coefficient $\sigma_{r}=4$ in the urban macro-cell with rich scattering environment, the new downlink capacity of multi-cell MISO cooperative cellular network with co-channel interference is given by

$$
C_{k}=\mathrm{B}_{\mathrm{w}} \log _{2}\left(1+\frac{\sum_{c b=1}^{N_{b}} \sum_{j=1}^{N_{t}^{c}} \frac{1}{r_{c b}^{4}}\left|z_{c b, j}\right|^{2}}{N_{0}+\sum_{b=1}^{\infty} \sum_{j=1}^{N_{t}} \frac{1}{r_{b}^{4}}\left|z_{b, j}\right|^{2}}\right) .
$$

In this paper, we focus on cell-edge users located in cooperative areas of multi-cell MISO cellular network. Therefore the distances $r_{c b}$ between cooperative BSs and cell-edge users can be approximatly treated as equivalent. Furthermore, the path loss between cooperative BSs and users is approximated as a constant. To simplify the notation, we define

$$
\eta^{\prime}=\frac{S_{d}^{\prime}}{S_{I}^{\prime}}=\frac{\sum_{c b=1}^{N_{b}} \sum_{j=1}^{N_{t}^{c}}\left|z_{c b, j}\right|^{2}}{\sum_{i=1}^{\infty} \sum_{j=1}^{N_{t}} \frac{1}{r_{i}^{4}}\left|z_{i, j}\right|^{2}} .
$$

Considering that the power of AWGN $N_{0}$ can be ignored compared to the power of the received interference $P^{R_{X}}[20]$, (18) can be concisely expressed as

$$
C_{k} \approx \mathrm{B}_{\mathrm{w}} \log _{2}\left(1+r_{c b}^{-4} \eta^{\prime}\right) .
$$

The Nakagami shaping factor $m$ is configured as $m=1$ for both desired signals and interfering signals. Each signal passing through the Nakagami fading channel is assumed to follow an i.i.d complex Gaussian distribution with zero mean and variance of 1 , i.e., $z_{c b, j} \sim C N(0,1)$. Thus, the PDF of aggregated expected signal $S_{d}^{\prime}$ can be simplified as

$$
f_{d}(x)=\frac{x^{N_{b} N_{t}^{c}-1} e^{-x / 2}}{\left(N_{b} N_{t}^{c}-1\right) ! 2^{N_{b} N_{t}^{c}}}, x>0 .
$$

On the other hand, the PDF of aggregated interference $S_{I}^{\prime}$ with complex channel coefficients has already been derived in (7a). We assume that the interference signal passes through the same channel condition of the desired signal, i.e. the Nakagami-m fading channels with path loss, thus the PDF of the aggregated interference $S_{I}^{\prime}$ can be further simplified as

$$
f_{P R x}(y)=\sqrt{\frac{\gamma^{2}}{2 \pi}} \frac{e^{-\gamma^{2} /(2 y)}}{y^{3 / 2}}, y>0
$$

with

$$
\gamma=\frac{2 \Gamma\left(\frac{3}{2}\right) \pi \lambda_{B S} \Gamma\left(N_{t}+\frac{1}{2}\right)}{\left(N_{t}-1\right) !}
$$

In this paper, the expected signal $S_{d}^{\prime}$ and the aggregated interference $S_{I}^{\prime}$ are assumed statistically independent. Therefore the PDF of $\eta^{\prime}$ is given by

$$
\begin{aligned}
f\left(\eta^{\prime}\right) & =\frac{\sqrt{\frac{\gamma^{2}}{2 \pi}}}{\left(N_{b} N_{t}^{c}-1\right) ! 2^{N_{b} N_{t}^{c}}} \eta^{\prime N_{b} N_{t}^{c}-1} \\
& \times \int_{0}^{\infty} \exp \left(-\frac{\gamma^{2}}{2 z}-\frac{\eta^{\prime} z}{2}\right) z^{v-1} \mathrm{~d} z .
\end{aligned}
$$

Based on the table of integral in [27], (23) can be simplified as

$$
f\left(\eta^{\prime}\right)=\frac{\gamma^{v+1} \sqrt{2 / \pi}}{\left(N_{b} N_{t}^{c}-1\right) ! 2^{N_{b} N_{t}^{c}}} \eta^{\frac{v-1}{2}} K_{v}\left(\gamma \sqrt{\eta^{\prime}}\right)
$$

with

$$
v=N_{b} N_{t}^{c}-\frac{1}{2} .
$$

Based on (24a)-(24b), the average channel capacity can be derived by calculating the expectation of (20). After normalizing the bandwidth $B_{w}=1$, the normalized downlink average capacity of multi-cell MISO cooperative cellular network with co-channel interference is derived as

$$
\begin{aligned}
\bar{C}_{\text {Aver }} & =\frac{C_{\text {Aver }}}{\mathrm{B}_{\mathrm{w}}} \\
& =p \int_{0}^{\infty} \log _{2}\left(1+r_{c b}^{-4} \eta^{\prime}\right) \eta^{\prime \frac{v-1}{2}} K_{v}\left(\gamma \sqrt{\eta^{\prime}}\right) \mathrm{d} \eta^{\prime}
\end{aligned}
$$

with

$$
p=\frac{\gamma^{v+1} \sqrt{2 / \pi}}{\left(N_{b} N_{t}^{c}-1\right) ! 2^{N_{b} N_{t}^{c}}}
$$

where $v$ and $\gamma$ are given by (24a) and (22b), respectively.

The integral in (25a) will cause some difficulties in the practical engineering calculation. To simplify the expression, we will utilize a special function, the so-called Meijer's Gfunction, which is defined as [43]

$$
\begin{aligned}
& G_{p, q}^{m, n}\left(x \mid \begin{array}{c}
a_{1}, \cdots a_{p} \\
b_{1}, \cdots b_{q}
\end{array}\right) \\
& =\frac{1}{2 \pi \Im} \int_{0}^{\infty} \frac{\prod_{j=1}^{m} \Gamma\left(b_{j}-s\right) \prod_{j=1}^{n} \Gamma\left(1-a_{j}+s\right)}{\prod_{j=m+1}^{q} \Gamma\left(1-b_{j}+s\right) \prod_{j=n+1}^{p} \Gamma\left(a_{j}-s\right)} x^{s} \mathrm{~d} s
\end{aligned}
$$

where $0 \leq m \leq q, 0 \leq n \leq p$, and $\Im=\sqrt{-1}$. The $\log _{2}(\cdot)$ and $K_{v}(\cdot)$ functions can be expressed as a special form of Meijer's G-function. So, (25a) can be expressed by a new form with Meijer's G-functions

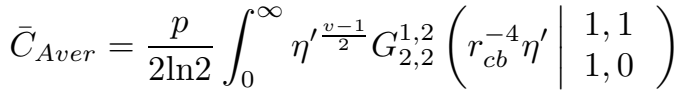

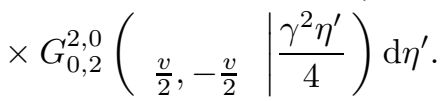

Based on the table of integral in [28], (25a) can be further simplified as follows by eliminating the integral calculation and parameter simplification

$$
\bar{C}_{\text {Aver }}=\frac{p}{2 \ln 2} r_{b}^{2(v+1)} G_{2,4}^{4,1}\left(\begin{array}{c|c}
-\frac{v+1}{2}, \frac{1-v}{2} \\
\left.-\frac{v}{2}, \frac{v}{2}, \frac{v+1}{2},-\frac{v+1}{2} \mid \frac{\gamma^{2} r_{b}^{4}}{4}\right)
\end{array}\right.
$$

where $p, v$ and $\gamma$ are given by (25b), (24a) and (22b), respectively. 


\section{Simulation Results and Discussions For Miso SySTEM CAPACITIES}

Based on the normalized downlink average capacity of the multi-cell MISO cellular network with co-channel interference derived in Section III, the effect of various system parameters on the capacity will be analyzed and compared by numerical calculations and MC simulations in this section. In what follows, some parameters of the capacity model are configured as follows: $\sigma_{d B}=7, m=1, \sigma_{r}=4, \sigma^{2}=1$, $\lambda_{B S}=1 /\left(\pi * 500^{2}\right)$, and $r_{c b}=500 \mathrm{~m}$. Every BS has no more than four antennas used for cooperative transmission, but in the default cooperative transmission scheme, every cooperative BS just uses two antennas. To simplify numerical analysis, the cooperative transmission among BSs is assumed as the aggregation of desired signals transmitted by the multi-antennas from different BSs, i.e., desired signals are accumulated directly by numerical calculation. The detailed cooperative transmission scheme, e.g., the joint pre-coding scheme [16], is not included in this paper for conciseness.

In Fig. 9, we first analyze the impact of the number of cooperative transmission (Co-Tx) antennas per cooperative BS on the normalized downlink average capacity. The number of cooperative BSs is noted as $C B S$. In numerical calculations, we obtain the following results. Without cooperative BSs, i.e., $C B S=1$, the capacity is improved by $209 \%$ when the number of Co-Tx antennas per cooperative BS is increased from 1 to 4 . With two cooperative BSs, i.e., $C B S=2$, the capacity is improved by $173 \%$ when the number of CoTx antennas per cooperative BS is increased from 1 to 4 . With three cooperative BSs, i.e., $C B S=3$, the capacity is improved by $153 \%$ when the number of Co-Tx antenna per cooperative BS is increased from 1 to 4 . When only one antenna per cooperative BS is used to transmit expected signals, the capacity is improved by $80.99 \%$ when CBS is increased from 1 to 2 ; the capacity is improved by $37.88 \%$ when CBS is increased from 2 to 3 . When four antennas per cooperative BS is used to transmit expected signals, the capacity is improved by $50.9 \%$ when CBS is increased from 1 to 2 ; the capacity is improved by $27.62 \%$ when CBS is increased from 2 to 3 . Therefore, with the increasing number of antennas or cooperative BSs, the capacity performance is improved, but the increment of capacity performance is decreased. Compared with results from MC simulations, these numerical results are validated.

In Fig. 10, the impact of the density parameter of interfering BSs $\lambda_{B S}$ on the normalized downlink average capacity is analyzed by numerical calculations and MC simulations. Based on numerical calculations, the following results are analyzed. Without cooperation BSs, i.e., $C B S=1$, the capacity decreases by $94.86 \%$ when the density parameter of interfering BSs is increased from $0.5 \times 10^{-6} \mathrm{~m}^{-2}$ to $3.5 \times 10^{-6} \mathrm{~m}^{-2}$. In the case of two cooperative BSs, i.e., $C B S=2$, the capacity decreases by $93.40 \%$ when the density parameter of interfering BSs is increased from $0.5 \times 10^{-6} \mathrm{~m}^{-2}$ to $3.5 \times 10^{-6} \mathrm{~m}^{-2}$. In the case of three cooperative BSs, i.e., $C B S=3$, the capacity decreases by $92.22 \%$ when the density parameter of interfering BSs is increased from $0.5 \times 10^{-6} \mathrm{~m}^{-2}$ to $3.5 \times 10^{-6} \mathrm{~m}^{-2}$. These results indicate that the density parameter of interfering BSs

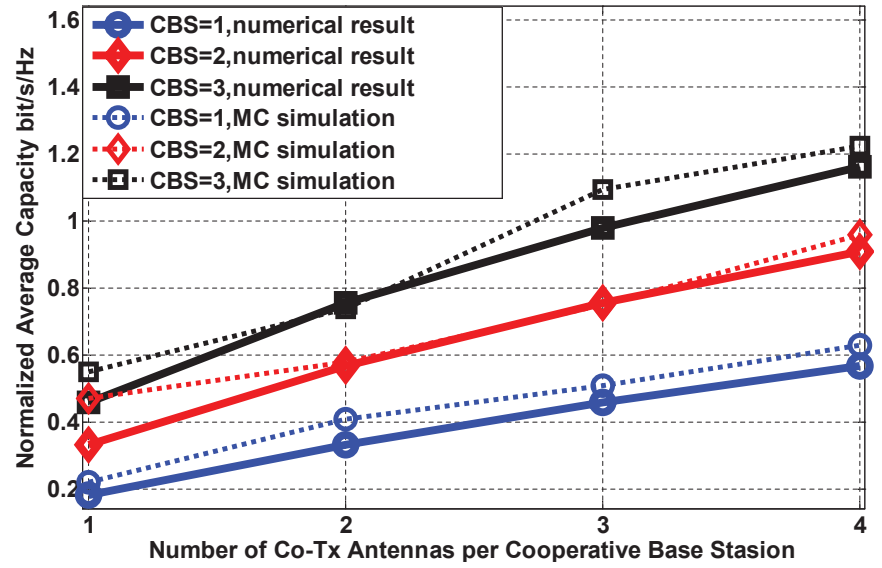

Fig. 9. Impact of the number of Co-Tx antennas per cooperative BS on the normalized downlink average capacity of multi-cell MISO cellular networks.

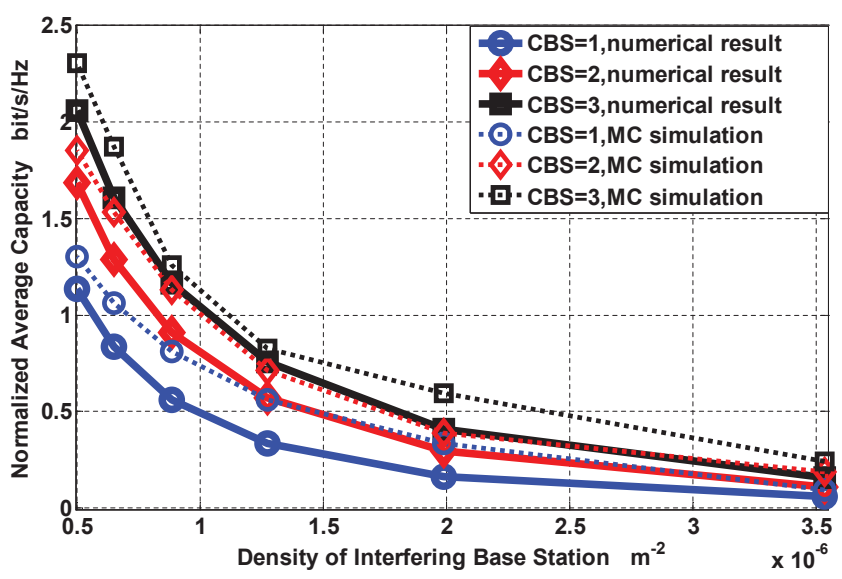

Fig. 10. Impact of the density of interfering BSs on the normalized downlink average capacity of multi-cell MISO cellular networks.

can obviously decrease the capacity performance in a multicell MISO cellular network. When the density parameter of interfering BSs is configured as $0.5 \times 10^{-6} \mathrm{~m}^{-2}$, compared with the capacity of one BS, the average capacity of two cooperative BSs improves by $48.76 \%$; compared with the average capacity of two cooperative BSs, the average capacity of three cooperative BSs improves by $21.99 \%$. Hence, with the specified density of interfering BSs, the cooperative transmission can improve the capacity performance, but this gain of capacity performance is decreased with the increasing number of cooperative BSs. When the density of interfering BSs is configured as $3.5 \times 10^{-6} \mathrm{~m}^{-2}$, compared with the capacity of one BS, the average capacity of two cooperative BSs improves by $90.98 \%$; compared with the average capacity of two cooperative BSs, the average capacity of three cooperative BSs improves by $43.81 \%$. Therefore, compared with the capacity performance in the small density of interfering BSs, the cooperative transmission can obviously improve the capacity performance with a high density of interfering BSs. From MC simulations, we can obtain consistent results with numerical calculations.

Fig. 11 shows the normalized downlink average capacity without and with AWGN, computed by means of numerical calculations and MC simulations, respectively. In the most 


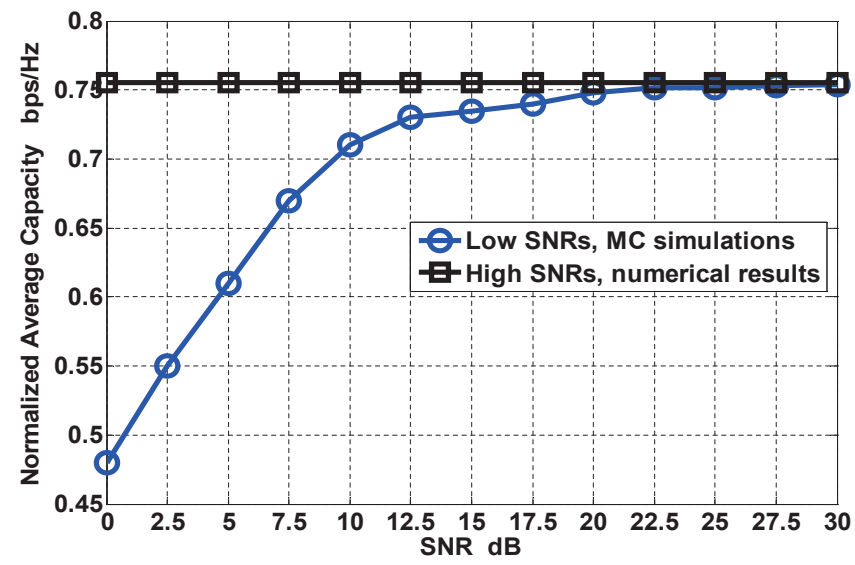

Fig. 11. Impact of the AWGN on the normalized downlink average capacity of multi-cell MISO cellular networks.

cases, the power of AWGN $N_{0}$ can be ignored comparing with the power of the received interference signal [20] in multi-cell MISO cellular networks. Based on this assumption, a closed-form normalized downlink average capacity of multicell MISO cooperative cellular networks i.e., (28) is derived. This result can be used in high signal-to-noise ratio (SNR) scenarios. However, in some special cases, the power of AWGN $N_{0}$ can not be ignored in multi-cell MISO cellular networks. Hence, the capacity performance with low SNR in multicell MISO cooperative cellular networks is analyzed by MC simulations. Based on default parameters in this section, we compare the normalized downlink average capacity without or with AWGN in Fig. 11. From Fig. 11, the AWGN has great impact on the normalized downlink average capacity when the SNR is less than or equal to $12.5 \mathrm{~dB}$; however, the AWGN has little impact on the normalized downlink average capacity when the SNR is larger than $12.5 \mathrm{~dB}$.

\section{Conclusions}

In this paper, we have derived the exact downlink average capacity of multi-cell MIMO cellular network with cochannel interference. Furthermore, the analytical closed-form normalized downlink average capacity for cell-edge users in a multi-cell MISO cooperative cellular network with cochannel interference has been derived and analyzed numerically. To derive this downlink capacity model, an analytical cochannel interference model has been proposed for multi-cell MIMO cellular networks. Based on the proposed closed-form normalized downlink average capacity of multi-cell MISO cooperative cellular network with co-channel interference, simulation results have shown that the cooperative transmission can improve the capacity performance in most cases, but the capacity gains diminish with the increasing number of cooperative BSs or antennas. Our analysis indicates that the cooperative transmission can efficiently enhance the capacity performance, especially in scenarios with high densities of interfering BSs. For our future work, we will explore the impact of different cooperative transmission schemes on the system capacity.

\section{APPENDIX A \\ DERIVATION OF (15)}

In this appendix, we derive the covariance of expected signals at the user $\mathrm{UE}_{k}$. In one cooperative cluster, one cell-edge user can receive expected signals and unexpected signals transmitted from different cooperative BSs. To simplify the complex of derivation, expected signals and unexpected signals from different cooperative BSs in the same cooperative cluster $c$ received by the user $\mathrm{UE}_{k}$ are assumed to be uncorrelated, which can be expressed as follows

$$
\mathbb{E}\left[\mathbf{x}_{c b, k, c}\left(\mathbf{x}_{c b^{\prime}, k, c}\right)^{H}\right]=0, \forall c b \neq c b^{\prime}
$$

where $\mathbb{E}(\cdot)$ is the expectation calculation operator, $\mathbf{x}_{c b, k, c}$ is the expected signal transmitted to the user $\mathrm{UE}_{k}$ by BS $c b$ in the cooperative cluster $c, \mathbf{x}_{c b^{\prime}, k, c}$ is the unexpected signal transmitted to the user $\mathrm{UE}_{k}$ by the $\mathrm{BS} c b^{\prime}$ in the cooperative cluster c. Moreover, the expected signal $\mathbf{x}_{c b, k, c}$ is assumed to have i.i.d. complex Gaussian entries with zero mean and unit variance. Therefore, the covariance of the expected signal $\mathbf{Q}_{c b, k, c}$ can be given by

$$
\mathbf{Q}_{c b, k, c}=\mathbb{E}\left[\mathbf{x}_{c b, k, c} \mathbf{x}_{c b, k, c}^{H}\right]=P_{\mathrm{ant}} \mathcal{I}
$$

where $\mathcal{I}$ is the unite matrix. Furthermore, in the scenario of multi-cell MISO cooperative cellular network, the signal covariance transmitted to the user $\mathrm{UE}_{k}$ by different BSs in the cooperative cluster $c$ can be expressed by

$$
\begin{aligned}
R_{x x} & =\mathbb{E}\left(\sum_{c b=1}^{N_{b}} \mathbf{h}_{c b, k, c} \mathbf{x}_{c b, k, c}\left(\mathbf{x}_{c b, k, c}\right)^{H}\left(\mathbf{h}_{c b, k, c}\right)^{H}\right) \\
& +\mathbb{E}\left(\sum_{c b=1}^{N_{b}} \sum_{\substack{c b^{\prime}=1 \\
c b^{\prime} \neq c b}}^{N_{b}} \mathbf{h}_{c b, k, c} \mathbf{x}_{c b, k, c}\left(\mathbf{x}_{c b^{\prime}, k, c}\right)^{H}\left(\mathbf{h}_{c b^{\prime}, k, c}\right)^{H}\right)
\end{aligned}
$$

where $\mathbf{h}_{c b, k, c}$ and $\mathbf{h}_{c b^{\prime}, k, c}$ are the channel matrices from the $\mathrm{BS} c b$ and BS $c b^{\prime}$ to the user $\mathrm{UE}_{k}$ in the cooperative cluster $c$, respectively. Base on (29) and (30), we only consider the Nakagami-m fading effect and (31) can be further simplified as

$$
\begin{aligned}
R_{x x} & =\mathbb{E}\left(\sum_{c b=1}^{N_{b}} \mathbf{h}_{c b, k, c} \mathbf{x}_{c b, k, c}\left(\mathbf{x}_{c b, k, c}\right)^{H}\left(\mathbf{h}_{c b, k, c}\right)^{H}\right) \\
& =\sum_{c b=1}^{N_{b}}\left[\mathbf{h}_{c b, k, c} \mathbf{Q}_{c b, k, c} \mathbf{h}_{c b, k, c}^{H}\right] \\
& =P_{a n t} \sum_{c b=1}^{N_{b}}\left\|\mathbf{h}_{c b, k, c}\right\|_{F}^{2} \\
& =P_{a n t} \sum_{c b=1}^{N_{b}} \sum_{j=1}^{N_{t}^{c}}\left|\mathbf{h}_{c b, j}\right|^{2} \\
& =P_{a n t} \sum_{c b=1}^{N_{b}} \sum_{j=1}^{N_{t}^{c}} \frac{1}{r_{c b}^{\sigma^{r}}}\left|z_{c b, j}\right|^{2} .
\end{aligned}
$$

This completes the derivation. 


\section{REFERENCES}

[1] C.-X. Wang, X. Hong, X. Ge, X. Cheng, G. Zhang, and J. S. Thompson, "Cooperative MIMO channel models: a survey," IEEE Commun. Mag., vol. 48, no. 2, pp. 80-87, Feb. 2010.

[2] I. Telatar, "Capacity of multi-antenna Gaussian channels," Eur. Trans. Telecommun., vol. 10, no. 6, pp. 585-595, Nov. 1999.

[3] S. Catreux, P. F. Driessen, and L. J. Greenstein, "Attainable throughput of an interference-limited multiple-input multipleoutput (MIMO) cellular system," IEEE Trans. Commun., vol. 49, no. 8, pp. 1307-1311, Aug. 2001.

[4] R. S. Blum, "MIMO capacity with interference," IEEE J. Sel. Areas Commun., vol. 21, no. 5, pp. 793-801, June 2003.

[5] E. S. Sousa, "Performance of a spread spectrum packet radio network link in a Poisson field of interferers," IEEE Trans. Inf. Theory, vol. 38, no. 6, pp. 1743-1754, Nov. 1992.

[6] X. Yang and A. Pertropulu, "Co-channel interference modeling and analysis in a Poisson field of interferers in wireless communications," IEEE Trans. Signal Process., vol. 51, no. 1, pp. 63-76, Jan. 2003.

[7] E. Salbaroli and A. Zanella, "Interference analysis in a Poisson field of nodes of finite area," IEEE Trans. Veh. Technol., vol. 58, no. 4, pp. 1776-1783, May 2009.

[8] M. Z. Win, P. C. Pinto, and L. A. Shepp, "A mathematical theory of network interference and its applications," Proc. IEEE, vol. 97, no. 2, pp. 205-230, Feb. 2009.

[9] K. Gulati, A. Chopra, B. L. Evans, and K. R. Tinsley, "Statistical modeling of co-channel interference," in Proc. IEEE Globecom, Nov. 2009, pp. 1-6.

[10] K. Gulati, B. L. Evans, and K. R. Tinsley, "Statistical modeling of cochannel interference in a field of Poisson distributed interferers," in Proc. IEEE ICASSP, Mar. 2010, pp. 3490-3493.

[11] K. Gulati, B. L. Evans, J. G. Andrews, and K. R. Tinsley, "Statistics of co-channel interference in a field of Poisson and Poisson-Poisson clustered interferers," IEEE Trans. Signal Process., vol. 58, no. 12, pp. 6207-6222, Dec. 2010.

[12] G. J. Foschini, Jr. and M. J. Gans, "On limits of wireless communication in a fading environment when using multiple antennas," Wireless Personal Commun., vol. 6, pp. 311-335, Mar. 1998.

[13] M. Webb, M. Beach, and A. Nix, "Capacity limits of MIMO channels with co-channel interference," in Proc. IEEE VTC'04-Spring, May 2004, pp. 703-707.

[14] Y. Wang and D. Yue, "Capacity of MIMO Rayleigh fading channels in the presence of interference and receive correlation," IEEE Trans. Veh. Technol., vol. 58, no. 8, pp. 4398-4405, Oct. 2009.

[15] V. S. Annapureddy, V. V. Veeravalli, and S. Vishwanath, "On the sum capacity of MIMO interference channels in the low interference regime," in Proc. IEEE Conf. Systems, Signals \& Computers, Nov. 2008, pp. 8084.

[16] J. S. Kwak, J. G. Andrews, and A. Lozano, "MIMO capacity in correlated interference-limited channels," in Proc. IEEE Symp. Inf. Theory, June 2007, pp. 106-110.

[17] M. Chiani, M. Z. Win, and H. Shin, "MIMO networks: the effects of interference," IEEE Trans. Commun., vol. 57, no. 4, pp. 1050-1060, Sep. 2008.

[18] S. Ye and R. S. Blum, "Some properties of the capacity of MIMO systems with co-channel interference," in Proc. IEEE ICASSP, Mar. 2005, pp. 1153-1156.

[19] Y. Chen, L. Hu, C. Yuen, Y. Zhang, Z. Zhang, and P. Rapajic, "Intrinsic measure of diversity gains in generalised distributed antenna systems with cooperative users," IET Commun., vol. 3, no. 2, pp. 209-222, Feb. 2009.

[20] Y. Chen, C. Yuen, and Y. H. Chew, "Double-directional information azimuth spectrum and relay network tomography for a decentralized wireless relay network," in Proc. IEEE ISITA, Oct. 2010, pp. 726-731.

[21] A. Tolli, M. Codreanu, and M. Juntti, "Cooperative MIMO-OFDM cellular system with soft handover between distributed base station antennas," IEEE Trans. Wireless Commun., vol. 7, no. 4, pp. 1428-1440, Apr. 2008.

[22] J. G. Andrews, F. Baccelli, and R. K. Ganti, "A new tractable model for cellular coverage," in Proc. IEEE Conf. Commun., Control, and Computing, Sep. 2010, pp. 1204-1211.

[23] F. Baccelli, B. Blaszczyszyn, and M. K. Karray, "Up- and downlink admission/congestion control and maximal load in large homogeneous CDMA networks," Mobile Networks and Applications, vol. 9, no. 6, pp. 605-617, June 2004.

[24] K. Huang, V. K. N. Lau, and Y. Chen, "Spectrum sharing between cellular and mobile ad hoc networks: transmission-capacity trade-off," IEEE J. Sel. Areas Commun., vol. 27, no. 7, pp. 1256-1267, July 2009.
[25] M. K. Simon and M. S. Alouini, Digital Communication over Fading Channels: A Unified Approach to Performance Analysis. Wiley, 2000.

[26] A. Goldsmith, Wireless Communications. Cambridge University Press, 2005.

[27] P. S. Bithas, N. C. Sagias, P. T. Mathiopoulos, G. K. Karagiannidis, and A. A. Rontogiannis, "On the performance analysis of digital communications over generalized-K fading channels," IEEE Commun. Lett., vol. 10, no. 5, pp. 353-355, May 2006.

[28] I. S. Gradshteyn and I. M. Ryzhik, Table of Integrals, Series, and Products. Academic Press, 2007.

[29] C.-X. Wang, X. Hong, H. Wu, and W. Xu, "Spatial temporal correlation properties of the 3GPP spatial channel model and the Kronecker MIMO channel model," EURASIP J. Wireless Commun. and Networking, vol. 2007, article ID 39871, 9 pages, 2007.

[30] M. Costa, "On the Gaussian interference channel," IEEE Trans. Inf. Theory, vol. 32, no. 31, pp. 607-615, Sep. 1985.

[31] Y. M. Shobowale and K. A. Hamdi, "A unified model for interference analysis in unlicensed frequency bands," IEEE Trans. Wireless Commun., vol. 8, no. 8, pp. 4004-4013, Aug. 2009.

[32] G. Samoradnitsky and M. Taqqu, Stable Non-Gaussian Random Processes. Chapman and Hall, 1994.

[33] J. P. Nolan, "Stable distributions: models for heavy tailed data," Commun. Stat.-Stochast. Models, pp. 56-215, May 1997.

[34] W. C. Y. Lee, "Estimate of channel capacity in Rayleigh fading environment," IEEE Trans. Veh. Technol., vol. 39, no. 3, pp. 187-189, Aug. 1990.

[35] A. A. Abu-Dayya and N. C. Beaulieu, "Outage probabilities of diversity cellular systems with cochannel interference in Nakagami fading," IEEE Trans. Veh. Technol., vol. 41, no. 4, pp. 343-355, Nov. 1992.

[36] D. Kim, M. A. Ingram, and W. W. Smith, "Measurements of small scale fading and path loss for long range RF tags," IEEE Trans. Antennas Propag., vol. 51, no. 8, pp. 1740-1749, Aug. 2003.

[37] G. K. Karagiannidis, "Moments-based approach to the performance analysis of equal gain diversity in Nakagami-m fading," IEEE Trans. Commun., vol. 52, no. 5, pp. 685-690, May 2004.

[38] C.-X. Wang, X. Hong, H.-H. Chen, and J. S. Thompson, "On capacity of cognitive radio networks with average interference power constraints," IEEE Trans. Wireless Commun., vol. 8, no. 4, pp. 1620-1625, Apr. 2009.

[39] X. Hong, C.-X. Wang, and J. S. Thompson, "Interference modelling of cognitive radio networks," in Proc. IEEE VTC'08-Spring, May 2008, pp. 1851-1855.

[40] T. K. Y. Lo, "Maximum ratio transmission," IEEE Trans. Commun., vol. 47, pp. 1458-1461, no. 10, Oct. 1999.

[41] P. A. Dighe, R. K. Mallik, and S. S. Jamuar, "Analysis of transmitreceive diversity in Rayleigh fading," IEEE Trans. Commun., vol. 51, no. 4, pp. 694-703, Apr. 2003.

[42] H. Dai and H. V. Poor, "Asymptotic spectral efficiency of multicell MIMO systems with frequency-flat fading," IEEE Trans. Signal Process., vol. 51, no. 11, pp. 2976-2988, Jan. 2003.

[43] Wolfram Mathworld, 2010. Available: http://functions.wolfram.com/HypergeometricFunctions.

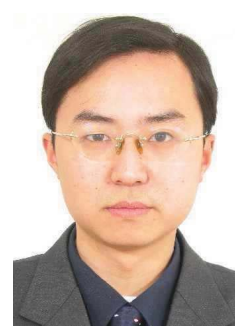

Xiaohu Ge (M'09-SM'11) is currently a Professor with the Department of Electronics and Information Engineering at Huazhong University of Science and Technology (HUST), China. He received his Ph.D. degree in Communication and Information Engineering from HUST in 2003. He has worked at HUST since Nov. 2005. Prior to that, he worked as an assistant researcher at Ajou University (Korean) and Politecnico Di Torino (Italy) from Jan. 2004 to Oct. 2005. He was a visiting researcher at Heriot-Watt University, Edinburgh, UK from June to August 2010. His research interests are in the area of mobile communications, traffic modeling in wireless networks, green communications, and interference modeling in wireless communications. He has published about 50 papers in refereed journals and conference proceedings and has been granted about 10 patents in China. He is leading several projects funded by NSFC, China MOST, and industries. He is taking part in several international joint projects, such as the RCUK funded UK-China Science Bridges: R\&D on (B) $4 \mathrm{G}$ Wireless Mobile Communications and the EU FP7 funded project: Security, Services, Networking and Performance of Next Generation IP-based Multimedia Wireless Networks. 
Dr Ge is currently serving as an Editor for International Journal of Communication Systems (John Wiley \& Sons) and KSII Transactions on Internet and Information Systems. Since 2005, he has been actively involved in the organisation of more than 10 international conferences, such as Publicity Chair of IEEE Europecomm 2011 and Co-Chair of workshop of Green Communication of Cellular Networks at IEEE GreenCom 2010. He is a Senior Member of the IEEE, a Senior member of the Chinese Institue of Electronics, a Senior member of the China Institute of Communications, and a member of the NSFC and China MOST Peer Review College.

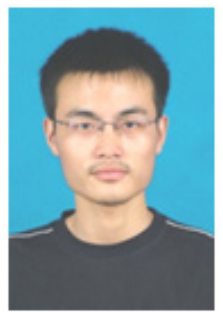

Kun Huang received the M.S. degree in Communication and Information Engineering form Huazhong University of Science and Technology (HUST), China. His current research interests are in the areas of interference modeling in multi-input multi-output (MIMO) antenna systems, algorithms of power control and muti-user scheduling in wireless systems.

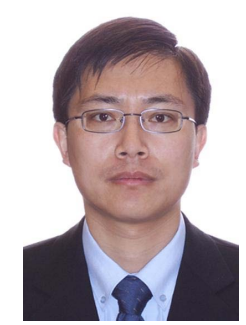

Cheng-Xiang Wang (S'01-M'05-SM'08) received the BSc and MEng degrees in Communication and Information Systems from Shandong University, China, in 1997 and 2000, respectively, and the Ph.D. degree in Wireless Communications from Aalborg University, Denmark, in 2004.

He has been with Heriot-Watt University, Edinburgh, UK, since 2005, first as a Lecturer, then as a Reader in 2009, and then as a Professor since 2011. $\mathrm{He}$ is also an Honorary Fellow of the University of Edinburgh, UK, a Chair Professor of Shandong University, a Guest Professor of Huazhong University of Science and Technology, an Adjunct Professor of Guilin University of Electronic Technology, and a Guest Researcher of Xidian University, China. He was a Research Fellow at the University of Agder, Grimstad, Norway, from 2001-2005, a Visiting Researcher at Siemens AG-Mobile Phones, Munich, Germany, in 2004, and a Research Assistant at Technical University of HamburgHarburg, Hamburg, Germany, from 2000-2001. His current research interests include wireless channel modeling and simulation, green communications, cognitive radio networks, vehicular communication networks, mobile femtocell networks, cooperative (relay) MIMO communications, and (beyond) 4G wireless communications. He has published 1 book chapter and more than 150 papers in refereed journals and conference proceedings. He is leading several projects funded by EPSRC, Mobile VCE, and industries, including the RCUK funded UK-China Science Bridges: R\&D on (B)4G Wireless Mobile Communications.
Dr Wang is currently serving as an Associate Editor for IEEE TRANSACTIONS ON VEHICULAR TECHNOLOGY and Editor for Wireless Communications and Mobile Computing Journal (John Wiley \& Sons) and Security and Communication Networks Journal (John Wiley \& Sons). He also served as an Editor for IEEE TRANSACTIONS ON WIRELESS COMMUNICATIONS (2007-2009) and Hindawi Journal of Computer Systems, Networks, and Communications (2007-2011). He was the leading Guest Editor for IEEE Journal on Selected Areas in Communications, Special Issue on Vehicular Communications and Networks. He served or is serving as a TPC member, TPC Chair, and General Chair for more than 60 international conferences. He received the IEEE Globecom'10 Best Paper Award in 2010 and the IEEE ICCT'11 Best Paper Awards in 2011. Dr Wang is listed in Dictionary of International Biography 2008 and 2009. Who's Who in the World 2008 and 2009, Great Minds of the 21st Century 2009, and 2009 Man of the Year. He is a Senior Member of the IEEE, a member of the IET, a Fellow of the HEA, and a member of EPSRC Peer Review College.

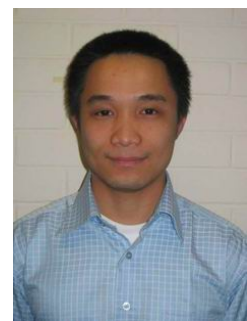

Xuemin Hong received his BSc degree in Communication Engineering from Beijing Information Science and Technology University, China, in 2004 and his $\mathrm{PhD}$ degree in Wireless Communications from Heriot-Watt University, UK, in 2008.

Since July 2011, he has been an Associate Professor with the School of Information Scinece and Technology, Xiamen University, China. From August 2009 to July 2011, he was a Post-doc Research Associate with the Joint Research Institute for Signal and Image Processing, Heriot-Watt University, UK. From January 2009 to July 2009, he was a Post-doc Research Fellow with the Department of Electrical and Computer Engineering, University of Waterloo, Canada. From 2004 to 2005, he was affiliated with the Centre for Telecommunication Research, King's College London, UK.

Dr Hong's research interests include MIMO and cooperative systems, ultrawideband (UWB) systems, wireless radio channel modelling, cognitive radio networks, and (beyond) 4G wireless communications. He has published more than 20 technical papers in major international journals and conferences and one book chapter in the area of wireless communications.

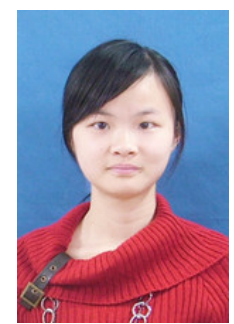

Xi Yang received the BS degree in electronic and information engineering from Huazhong University of Science and Technology (HUST), China, in June 2010. She is currently working toward the MS degree in communication and information systems at HUST. Her research interests include multi-user MIMO techniques and interference modeling in wireless communications. 